(W)

Check for

Cite as

Nano-Micro Lett.

(2021) 13:206

Received: 29 June 2021

Accepted: 23 August 2021

Published online: 11 October 2021

(C) The Author(s) 2021

\section{D Ultralight Hollow NiCo Compound@MXene Composites for Tunable and High-Efficient Microwave Absorption}

\author{
Hui-Ya Wang ${ }^{1}$, Xiao-Bo Sun ${ }^{1}$, Shu-Hao Yang ${ }^{1}$, Pei-Yan Zhao ${ }^{1}$, Xiao-Juan Zhang ${ }^{2}$, \\ Guang-Sheng Wang ${ }^{\circledR}$, Yi Huang ${ }^{3}$
}

\title{
HIGHLIGHTS
}

- Ultralight 3D NiCo compound@MXene nanocomposites that inherited hollow polyhedral skeleton and excellent conductive network were fabricated.

- Excellent electromagnetic absorption performance was achieved with optimal RLmin value of - 67.22 dB and ultra-wide EAB of $6.72 \mathrm{GHz}$ under the low filler loading.

- Electromagnetic parameters and microwave absorption property can be distinctly or slightly regulated by adjusting the filler loading and decoration of $\mathrm{Ti}_{3} \mathrm{C}_{2} \mathrm{~T}_{\mathrm{x}}$ nanoflakes.

ABSTRACT The 3D hollow hierarchical architectures tend to be designed for inhibiting stack of MXene flakes to obtain satisfactory lightweight, high-efficient and broadband absorbers. Herein, the hollow NiCo compound@MXene networks were prepared by etching the ZIF 67 template and subsequently anchoring the $\mathrm{Ti}_{3} \mathrm{C}_{2} \mathrm{~T}_{x}$ nanosheets through electrostatic selfassembly. The electromagnetic parameters and microwave absorption property can be distinctly or slightly regulated by adjusting the filler loading and decoration of $\mathrm{Ti}_{3} \mathrm{C}_{2} \mathrm{~T}_{x}$ nanoflakes. Based on the synergistic effects

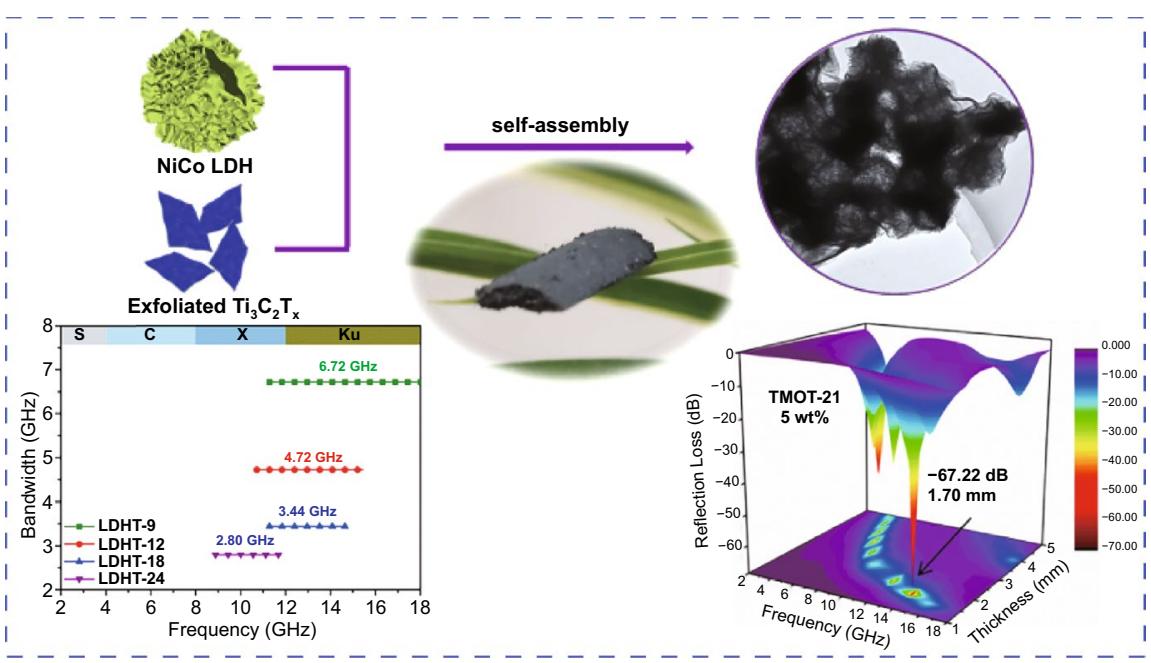
of multi-components and special well-constructed structure, NiCo layered double hydroxides @ $\mathrm{Ti}_{3} \mathrm{C}_{2} \mathrm{~T}_{x}(\mathrm{LDHT}-9)$ absorber remarkably achieves unexpected effective absorption bandwidth (EAB) of $6.72 \mathrm{GHz}$ with a thickness of $2.10 \mathrm{~mm}$, covering the entire Ku-band. After calcination, transition metal oxide @ $\mathrm{Ti}_{3} \mathrm{C}_{2} \mathrm{~T}_{x}$ (TMOT-21) absorber near the percolation threshold possesses minimum reflection loss ( $\mathrm{RL}_{\text {min }}$ ) value of $-67.22 \mathrm{~dB}$ at $1.70 \mathrm{~mm}$ within a filler loading of only $5 \mathrm{wt} \%$. This work enlightens a simple strategy for constructing MXene-based composites to achieve high-efficient microwave absorbents with lightweight and tunable EAB.

KEYWORDS 3D hollow hierarchical architecture; Tunable EAB; High-performance microwave absorption; Ultralight

Xiao-Juan Zhang, zhxiaojuan@btbu.edu.cn; Guang-Sheng Wang, wanggsh@buaa.edu.cn

School of Chemistry, Beihang University, Beijing 100191, People's Republic of China

2 College of Chemistry and Materials Engineering, Beijing Technology and Business University, Beijing 100048, People's Republic of China

3 School of Materials Science and Engineering, Nankai University, Tianjin 300350, People's Republic of China 


\section{Introduction}

Currently, the burgeoning 5G telecommunication is a newgeneration technology with faster transmission speed, larger network capacity and shorter delay. However, it cannot be ignored the growing hazard of the ensuing electromagnetic interference (EMI) and electromagnetic (EM) radiation, which directly affects human physical health and normal operation of electronic devices. Hence, the intense demands for suppression of harm EM pollution/radiation accelerate the research on high-performance microwave absorption (MA) materials in the $\mathrm{GHz}$ range [1-3].

Over the past few decades, considerable efforts have been focused on selecting the proper components and structures. Generally, two-dimensional (2D) materials such as graphene [4], $\mathrm{g}_{-} \mathrm{C}_{3} \mathrm{~N}_{4}$ [5], and MXene [6] exhibit more in-plane reflection and multiple reflections between the layers as well as superior in-plane electron transfer, benefiting to promote the attenuation of EM wave [7]. Lately, transition metal carbides and nitrides (MXene), as a typical and novel 2D material, have garnered increasing attention and expansive application prospects in both EMI shielding and MA field owing to its extraordinary inherent conductivity, large specific surface area, superior mechanical properties and accordionlike morphology $[8,9]$. Besides, rich surface functional group, interfaces and intrinsic defects would contribute to the increase in dipole and interface polarization which are beneficial to form dielectric loss, making MXene become a promising candidate for EM absorbing materials [10].

However, the 2D merits of MXene are still hindered by its self-restacking or aggregation on accounted of strong van der Waals interaction and hydrogen bond between adjacent nanosheets [11]. It is not conducive to the full manifestation of surface active sites, multiple reflection and scattering of electromagnetic wave, which further result in obvious decrease in MA ability. Introducing interlayer spacers, such as metal ions [12], metal oxide nanoparticles [13], polymers [14] or constructing 3D hollow architecture, has been proved to be effective strategies to resolve this issue $[15,16]$. $\mathrm{Li}$ et al. [17] introduced Ni nanoparticles into multilayered gaps of the adjacent 2D MXene, which efficiently alleviated the self-restacking of MXene and displayed the distinct absorption property with reflection loss of $-50.5 \mathrm{~dB}$. In addition, the 2D MXene nanosheets enable to be constructed into special 3D structures via mechanical shearing [18], template method [19], self-assembly [20], spray drying [7] and so on. Zhao et al. [19] demonstrated on processing poly(methyl methacrylate) (PMMA) spherical as sacrificial templates to construct 3D macroporous frameworks. The results indicated these 3D MXene films exhibited much improved performance compared to multilayer MXenes. Liang et al. [2] prepared Ni/MXene hybrids via co-solvothermal method, which displayed a strong reflection loss of $-52.6 \mathrm{~dB}$ and broad EAB of 3.7 GHz. Cui et al. [7] successfully fabricated 3D pleated RGO/MXene/ $\mathrm{Fe}_{3} \mathrm{O}_{4}$ microspheres (FMCM) by ultrasonic spray technology. Benefiting from the $3 \mathrm{D}$ porous structure and reasonable composition, the FMCM composites showed excellent MA performance that the minimum reflection loss reached $-51.2 \mathrm{~dB}$ and $\mathrm{EAB}$ was $4.7 \mathrm{GHz}$ at $2.9 \mathrm{~mm}$. Although these structural engineering strategies are highly efficient, most of them are complicated with sophisticated post-processing or harsh preparation conditions, which easily induce oxidation and structural degradation [21]. Considering the device dependency and the integrity of MXene, it is an undoubtedly convenient and operable strategy to construct 3D hierarchical architecture by electrostatic self-assemble negatively charged MXene with suitable components.

Layered double hydroxides (LDHs), as a kind of lamellar material mixed with adjustable hydroxides containing divalent and trivalent metal ions, possess more abundant active sites than those of single metal compounds, which is helpful to achieve potentially valuable MA materials [22]. Furthermore, the LDHs tend to avoid skin effect derived from metalbased absorbents at high frequency and be favorable for the entry of EM waves due to their semiconductor characteristic. Compared with pure metal materials, the LDHs own better chemical stability to enlarge their practical applications [23]. Recently, metal organic frameworks (MOFs) are utilized as a promising self-template to construct $\mathrm{LDH}$ materials with hollow or core-shell structure, which normally equip with large surface areas and porous architecture to alleviate the stress and endow their enhanced MA performance [24, 25]. However, LDH monomers usually deliver limited local microcurrent networks, which directly result in poor conduction loss. Coupling with highly conductive materials is undoubtedly a feasible solution. For example, Zhao et al. [26] successfully synthesized CoNi@ NCPs-rGO composites 
by pyrolyzing the ZIF-67@CoNi LDHs-GO precursor. The optimal $\mathrm{RL}_{\min }$ of the compounds could reach up to $-58.2 \mathrm{~dB}$ and the $\mathrm{EAB}$ was $4.03 \mathrm{GHz}$ at $2.5 \mathrm{~mm}$ with a filler loading of $30 \%$. Wen et al. [23] prepared porous layered double hydroxide@carbon (LDH@C) composites through calcination and alkali treatment of $\mathrm{Co}_{x} \mathrm{Al}_{y}-\mathrm{MOF}-74$. The existence of polarization loss, electrical loss and open void space contributed to substantially strong $\mathrm{RL}_{\min }$ of $-45.7 \mathrm{~dB}$ and broaden $\mathrm{EAB}$ of $9.0 \mathrm{GHz}$ at $3.7 \mathrm{~mm}(20 \mathrm{wt} \%)$. Wang et al. [27] constructed carbon fabric interconnected with $\mathrm{NiFe}$ LDH/MXene-derived $\mathrm{FeNi}_{3} / \mathrm{TiO}_{2}$ absorber, and adjust the content of $\mathrm{FeNi}_{3} / \mathrm{TiO}_{2}$ surrounding the percolation threshold to achieve superior absorption performance. Under the addition of $7.0 \mathrm{wt} \% \mathrm{NiFe} \mathrm{LDH} / \mathrm{MXene}$ precursor, $\mathrm{FeNi}_{3} / \mathrm{TiO}_{2}$ displayed an optimal $\mathrm{RL}_{\min }$ of $-58.0 \mathrm{~dB}$ and a broad $\mathrm{EAB}$ of $7.0 \mathrm{GHz}$ at $2.5 \mathrm{~mm}$. However, it is relatively rare that $3 \mathrm{D}$ hollow LDH nanocages derived from MOF combine with MXene to release self-restacking and further optimize EM wave absorption.

Herein, we designed a facile and controllable strategy to construct 3D hollow NiCo LDH@ $\mathrm{Ti}_{3} \mathrm{C}_{2} \mathrm{~T}_{x}$ MXene (LDHT) network with hierarchical structure by electrostatical selfassembly. The existence of 3D hollow NiCo LDH with lowconductivity could not only alleviate the skin effect efficiently, but also weaken the self-restacking of MXene nanosheets simultaneously. Moreover, large interflake cavity also dramatically promotes the impedance matching character and prolongs the propagation path of electromagnetic wave to enhance the dielectric loss. Based on the superiority of the unique structure and the synergy effects of multiple components, the LDHT absorbents with different contents of MXene exhibit outstanding MA capabilities. It is found that the $\mathrm{RL}_{\text {min }}$ values gradually shift to the lower frequency accompanied with reduction in $\mathrm{EAB}$ as increasing the decoration of $\mathrm{Ti}_{3} \mathrm{C}_{2} \mathrm{~T}_{x}$ nanoflakes and filler loading in polyvinylidene fluoride (PVDF). In addition, regulating the mass of immobilized $\mathrm{Ti}_{3} \mathrm{C}_{2} \mathrm{~T}_{x}$ flakes can subtly tune the absorbing property compared with adjusting filler content. For comparison, NiCo transition metal oxide (TMO)@ $\mathrm{Ti}_{3} \mathrm{C}_{2} \mathrm{~T}_{x}$ MXene (TMOT) with fine adjustment of adherent $\mathrm{Ti}_{3} \mathrm{C}_{2} \mathrm{~T}_{x}$ MXene content were prepared by calcination to rationally manipulate the electromagnetic parameters as well as MA performance. This work highlights the optimized hollow framework structure of MOF-derived LDH anchored with MXene nanosheets and provides recent progress to construct MXene-based absorbers.

\section{Experimental Section}

\subsection{Materials}

$\mathrm{Ti}_{3} \mathrm{AlC}_{2}$ powders (400 mesh) were purchased from 11 Technology Co., Ltd (Jilin, China). Lithium fluoride (LiF), 2-Methylimidazole $\left(\mathrm{C}_{4} \mathrm{H}_{6} \mathrm{~N}_{2}\right)$, nickel nitrate hexahydrate $\left(\mathrm{Ni}\left(\mathrm{NO}_{3}\right)_{2} \cdot 6 \mathrm{H}_{2} \mathrm{O}\right)$ and cobalt nitrate hexahydrate $\left(\mathrm{Co}\left(\mathrm{NO}_{3}\right)_{2} \cdot 6 \mathrm{H}_{2} \mathrm{O}\right)$ were purchased from Macklin Co., Ltd (Shanghai, China). Hydrochloric acid $(\mathrm{HCl}, 37 \%), \mathrm{N}, \mathrm{N}-$ dimethylformamide (DMF), anhydrous ethanol $\left(\mathrm{C}_{2} \mathrm{H}_{5} \mathrm{OH}\right)$ and anhydrous methanol $\left(\mathrm{CH}_{3} \mathrm{OH}\right)$ wereobtained from Modern Oriental (Beijing) Technology Development Co., Ltd (Beijing China). All reagents were of analytical grade and used without further purification.

\subsection{Preparation of $\mathrm{Ti}_{3} \mathrm{C}_{2} \mathrm{~T}_{x}$ MXene Nanosheets}

Typically, $1.0 \mathrm{~g}$ of $\mathrm{LiF}$ was dispersed in $\mathrm{HCl}$ aqueous solution $\left(9 \mathrm{M}, 20 \mathrm{~mL}\right.$ ), followed by the slowly adding $1.0 \mathrm{~g} \mathrm{Ti}_{3} \mathrm{AlC}_{2}$ within $5 \mathrm{~min}$. After etching for $24 \mathrm{~h}$ at $35^{\circ} \mathrm{C}$ under continuous stirring, the product was washed with deionized water and then centrifuged at $3500 \mathrm{rpm}$ several times until the $\mathrm{pH}$ of 6 . Afterward, the precipitate was ultrasonicated for $90 \mathrm{~min}$ under Ar flow and centrifugated for $1 \mathrm{~h}$ at $3500 \mathrm{rpm}$. Finally, a dark green $\mathrm{Ti}_{3} \mathrm{C}_{2} \mathrm{~T}_{x}$ supernatant was collected.

\subsection{Preparation of Hollow NiCo LDH Nanocages}

The ZIF 67 polyhedrons were synthesized according to the reported method [28]. Firstly, $0.32 \mathrm{~g}$ of as-prepared ZIF 67 precursor and $0.6 \mathrm{~g}$ of $\mathrm{Ni}\left(\mathrm{NO}_{3}\right)_{2} \cdot 6 \mathrm{H}_{2} \mathrm{O}$ were dispersed into $60 \mathrm{~mL}$ of ethanol, respectively. After mixing the two solutions, the reaction vessel was treated at $90{ }^{\circ} \mathrm{C}$ for $80 \mathrm{~min}$. Subsequently, the hollow NiCo LDH nanocages can be collected by washing and centrifugation for several times with ethanol.

\subsection{Preparation of 3D Hollow NiCo LDH@ $\mathrm{Ti}_{3} \mathrm{C}_{2} \mathrm{~T}_{x}$ and NiCo TMO@ $\mathrm{Ti}_{3} \mathrm{C}_{2} \mathrm{~T}_{x}$ MXene Composites}

The hierarchical NiCo LDH@ $\mathrm{Ti}_{3} \mathrm{C}_{2} \mathrm{~T}_{x}$ (LDHT) nanocomposites were prepared through an electrostatic selfassemble process. Initially, $0.03 \mathrm{~g}$ of $\mathrm{NiCo} \mathrm{LDH}$ was uniformly dispersed in $20 \mathrm{~mL}$ of deionized water, and $\mathrm{Ti}_{3} \mathrm{C}_{2} \mathrm{~T}_{x}$ 
nanosheets suspension $\left(1.0 \mathrm{mg} \mathrm{mL}^{-1}\right)$ were added. After stirring for $20 \mathrm{~min}$, LDHT composites were obtained after freeze-drying, while NiCo TMO@ $\mathrm{Ti}_{3} \mathrm{C}_{2} \mathrm{~T}_{x}$ (TMOT) composites were fabricated by calcining LDHT composites at $350{ }^{\circ} \mathrm{C}$ under Ar atmosphere for $2 \mathrm{~h}$ with heating rate of $2{ }^{\circ} \mathrm{C} \min ^{-1}$. The resultant composites were denoted as LDHT-x and TMOT-x, where $\mathrm{x}(6,9,12$ 15, 18, 21, and 24) was the mass of $\mathrm{Ti}_{3} \mathrm{C}_{2} \mathrm{~T}_{x}$ MXene suspension.

\subsection{Characterization}

The crystal structure of the obtained products was identified by X-ray diffraction (XRD) pattern (Bruker D8 AVANCE X-ray diffractometer with $\mathrm{Cu} \mathrm{K} \alpha$ ). The morphologies and nanostructures were observed with field emission scanning electron microscopy (SEM, JSM-7500F) and transmission electron microscopy (TEM, JEM-2100F). X-ray photoelectron spectroscopy (XPS) was characterized by Thermo Escalab 250Xi X-ray photoelectron spectrometer to analyze the surface chemical compositions. The zeta potential was conducted on Malvern Zetasizer Nano ZS (Malvern Instruments, USA). Nitrogen adsorption/ desorption isotherms and pore structures of as-obtained samples were carried out by a Micromeritics ASAP 2460 analyzer. Atomic force microscopy (AFM) image was investigated by Bruker Dimension ICON with Nanoscope $\mathrm{V}$ controller. To measure the EM parameters, resultant products were mixed with PVDF in different mass percentages to be pressed into circular rings $\left(\Phi_{\text {out }}=7.00 \mathrm{~mm}\right.$ and $\Phi_{\text {in }}=3.04 \mathrm{~mm}$ ). Subsequently, it was tested with coaxial line method by a vector network analyzer (VNA; Agilent TE5071C).

\section{Results and Discussion}

\subsection{Morphological Structures and Phase Crystallinity of LDHT and TMOT}

The preparation procedure and proposed structure of the novel 3D hollow LDHT and TMOT are shown in Fig. 1. The LDHT nanocages have been synthesized by utilizing ZIF-67 precursor as template and subsequent electrostatically self-assemble process. To obtain bimetallic NiCo LDH, the ZIF-67 templates are gradually etched by the $\mathrm{H}^{+}$protons, meanwhile the $\mathrm{Co}^{2+}$ enable to be partially oxidized into $\mathrm{Co}^{3+}$ by the $\mathrm{NO}_{3}{ }^{-}$and dissolved oxygen. Finally, the co-precipitation of both $\mathrm{Co}^{2+} / \mathrm{Co}^{3+}$ and $\mathrm{Ni}^{2+}$ facilitates the formation of the hollow NiCo LDH nanocages [29, 30]. Moreover, the LDHT nanocages can be transformed into TMOT nanocages after calcination treatment.

Briefly, exfoliated $\mathrm{Ti}_{3} \mathrm{C}_{2} \mathrm{~T}_{x}$ MXene nanosheets are successfully achieved by selectively etching the $\mathrm{Al}$ layers of the pristine bulk $\mathrm{Ti}_{3} \mathrm{AlC}_{2}$. The as-obtained $\mathrm{Ti}_{3} \mathrm{C}_{2} \mathrm{~T}_{x}$ flakes have lateral size of 0.4-2.9 $\mu \mathrm{m}$ and thickness of $1.6 \mathrm{~nm}$ (Figs. 2a, c and S1a). High-resolution TEM (HRTEM, Fig. 2b) image reveals the distinct lattice fringes of 0.321 and $0.211 \mathrm{~nm}$, corresponding to the (0008) and (0012) plane of $\mathrm{Ti}_{3} \mathrm{C}_{2} \mathrm{~T}_{x}$ MXene. As shown in Fig. 2d, g, ZIF-67 precursors exhibit a rhombic dodecahedral structure with smooth surface and relatively uniform size of $700-800 \mathrm{~nm}$. The average size of $\mathrm{NiCo} \mathrm{LDH}$ is estimated approximately to be $850 \mathrm{~nm}$, inheriting the polyhedral skeleton structure of ZIF 67 , whose surface is constructed by a large number of ultrathin nanosheets (Fig. 2e). In addition, the enlarged SEM image (inset of Fig. 2e) and TEM image (Fig. 2h) clearly illustrate that the NiCo LDH particles display an inner hollow structure. Such porous and hollow nanocages composed of interconnected nanosheets endow large quantities of interfaces and heterojunctions, which are favorable to polarization and scattering of EM wave [31]. As shown in Fig. 2i, the $\mathrm{Ti}_{3} \mathrm{C}_{2} \mathrm{~T}_{x}$ nanosheets are negatively charged with a zeta potential of $-13.2 \mathrm{mV}$ derived from abundant surface functional groups $(-\mathrm{O},-\mathrm{OH}$, and $-\mathrm{F})$ caused by etching treatment, which endow MXenes with the capability to electrostatically decorate on $\mathrm{NiCo} \mathrm{LDH}(+23.34 \mathrm{mV})$. Interestingly, large flocculation appears and settles down to the bottom of the container as introducing hollow NiCo LDH nanocages into MXene solution, indicating the electrostatic self-assembly

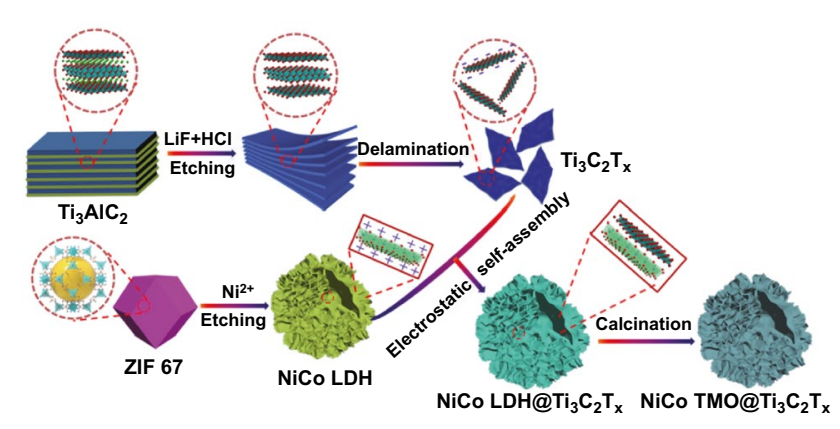

Fig. 1 Schematic diagram of synthetic process for LDHT and TMOT 


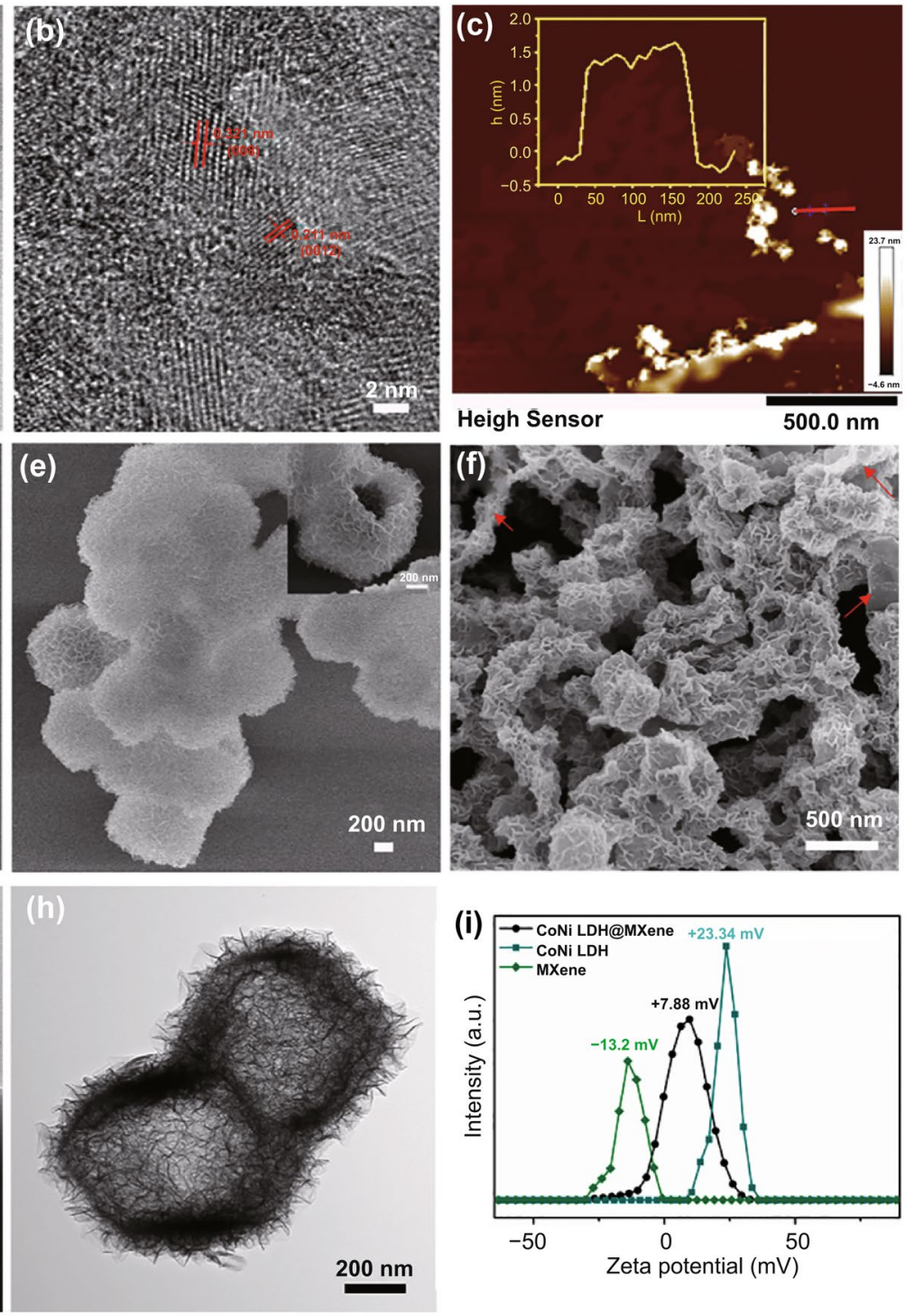

(i) $\rightarrow-$ CoNi LDH@MXene $+23.34 \mathrm{mV}$

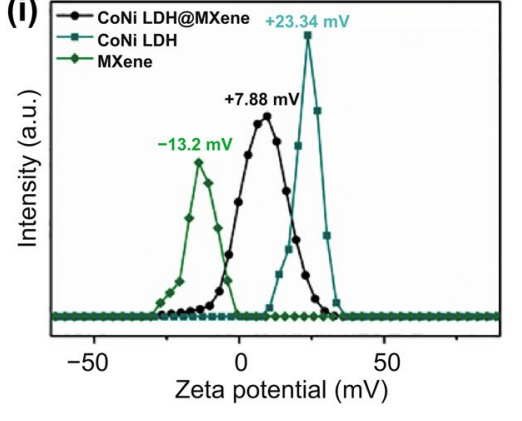

Fig. 2 a TEM, b HRTEM, c AFM images of $\mathrm{Ti}_{3} \mathrm{C}_{2} \mathrm{~T}_{x}$ nanosheets. $\mathbf{d}$ SEM, $\mathbf{g}$ TEM images of ZIF-67 precursors. e SEM, $\mathbf{h}$ TEM images of NiCo LDH nanocage. $f$ SEM image of hollow LDHT nanostructure. $i$ Zeta potentials image of $\mathrm{Ti}_{3} \mathrm{C}_{2} \mathrm{~T}_{x}$ suspension, NiCo LDH and LDHT

between NiCo LDH and MXene. The as-synthesized LDHT composites with a Zeta potential of $+7.88 \mathrm{mV}$ (Fig. 2i) emerge more clearly defined wrinkles on the surface and are connected to each other by thin MXene flakes marked with red arrows (Fig. 2f).

This hollow hierarchical architecture is further illustrated by TEM and HRTEM. As shown in Fig. 3, the smaller $\mathrm{Ti}_{3} \mathrm{C}_{2} \mathrm{~T}_{x}$ MXene nanosheets modify the surface of the hollow LDH/TMO structure, while the larger MXene nanosheets act as a connecting bridge, which is beneficial to the construction of the conductive network (Fig. 3a-c). In addition, TMOT products basically maintain the hollow polyhedron structure yet with slight collapse after calcination treatment (Fig. 3b). Two kinds of pronounced lattice fringes with lattice spacing of $0.209 \mathrm{~nm}$ and $0.259 \mathrm{~nm}$ are shown in Fig. 3d, corresponding to (0012) and (009) plane of $\mathrm{Ti}_{3} \mathrm{C}_{2} \mathrm{~T}_{x}$ and NiCo LDH, respectively [32]. The selected area electron diffraction (SAED) pattern (inset in Fig. 3d) exhibits several rings, confirming the poly-crystalline structure of LDHT composites. For TMOT samples (Fig. 3e), lattice fringes of $0.261,0.242$, and $0.213 \mathrm{~nm}$ can be ascribed to the (10 10 ), (311), and (200) plane of $\mathrm{Ti}_{3} \mathrm{C}_{2} \mathrm{~T}_{x}, \mathrm{NiCo}_{2} \mathrm{O}_{4}$, and $\mathrm{CoO}$, respectively [33-35]. Moreover, the well-defined rings shown in the SAED image manifest the same results. The 

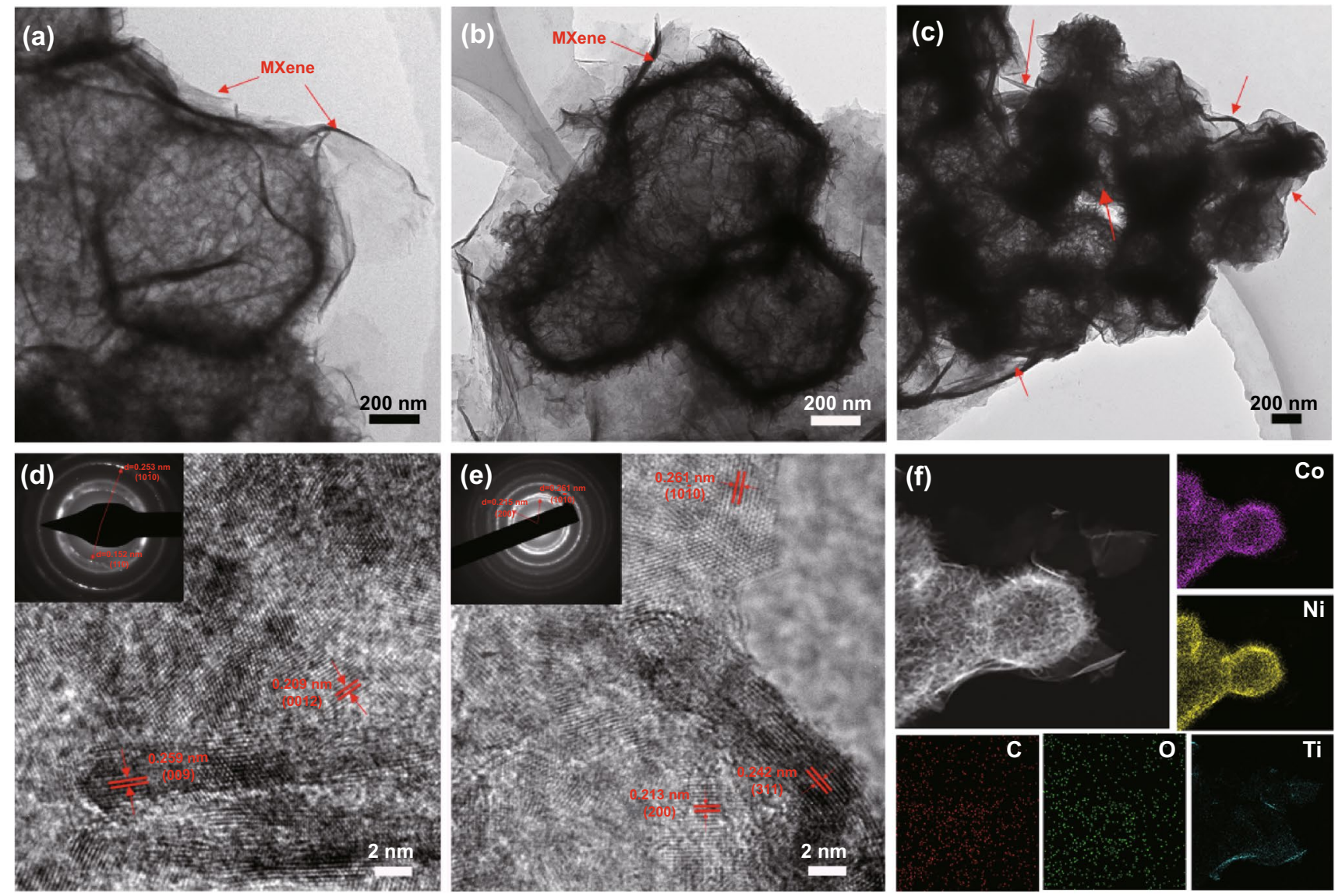

Fig. 3 a TEM and $\mathbf{d}$ HRTEM images of LDHT. b, $\mathbf{c}$ TEM e HRTEM, and $\mathbf{f}$ element mapping images of TMOT

HRTEM images and the corresponding SAED images show LDHT/TMOT with good crystallinity. Besides, the element mapping analysis shown in Fig. $3 f$ further demonstrates the uniform distribution of $\mathrm{Co}, \mathrm{Ni}, \mathrm{Ti}, \mathrm{C}$, and $\mathrm{O}$, which reveal the MXene successfully assemble on the surface of TMO. Besides, the obtained LDHT flocculation with the bulk density of $0.0484 \mathrm{~g} \mathrm{~cm}^{-3}$ can stand on a leaf well, proving it is an ultralight material (Fig. 4).

The composition and structural characteristics of the as-obtained samples are identified by XRD. As shown in Fig. 5a, the disappearance of the most intense diffraction peak at about $39^{\circ}$ in the $\mathrm{Ti}_{3} \mathrm{C}_{2} \mathrm{~T}_{x}$ MXene pattern indicates that $\mathrm{Al}$ layers are selectively removed. $\mathrm{Ti}_{3} \mathrm{C}_{2} \mathrm{~T}_{x}$ nanosheets display six peaks at $7.06^{\circ}, 14.33^{\circ}, 21.21^{\circ} 28.75^{\circ}, 36.12^{\circ}$, and $43.90^{\circ}$, which are assigned to the (0002), (0004), (0006), (0008), (0010), and (0012) crystal planes, respectively [36]. Moreover, the (0002) diffraction peak shifts to a lower angle in comparison with that of $\mathrm{Ti}_{3} \mathrm{AlC}_{2}$, proving the enlarged interlayer distance of the exfoliated $\mathrm{Ti}_{3} \mathrm{C}_{2} \mathrm{~T}_{x}$ nanosheets [37].
The XRD pattern of ZIF 67 (Fig. S2) matches well with the previous pattern [38]. For NiCo LDH sample shown in Fig. $5 \mathrm{~b}$, the main peaks at $10.37^{\circ}, 23.13^{\circ}, 33.67^{\circ}$, and $59.90^{\circ}$ are corresponding to the (003), (006), (009), and (110) planes of the typical LDH [39]. After electrostatic selfassembly, the new peak at $5.84^{\circ}$ corresponding to (0002) represents the successful combination of NiCo LDH and $\mathrm{Ti}_{3} \mathrm{C}_{2} \mathrm{~T}_{x}$ MXene nanosheets. The further increased interlayer spacing strongly suggests that the 3D hollow structure of the NiCo LDH efficiently alleviates the self-restacking of MXene nanosheets, which in turn enriches interfaces and prolongs the propagation path of EM wave. As for TMOT compounds (Fig. 5b), the diffraction peaks at $36.8^{\circ}$ and $44.5^{\circ}$ belong to the (311) and (400) planes of $\mathrm{NiCo}_{2} \mathrm{O}_{4}$ spinel phase (PDF No. 20-0781), respectively [30, 40]. Meanwhile, the diffraction peaks of $42.7^{\circ}$ and $60.8^{\circ}$ are associated with the (200) and (220) planes of $\mathrm{CoO}$ (PDF No. 71-1178), respectively. 




Fig. 4 Images of LDHT composite on a leaf and $\mathbf{b}$ reed flocculants
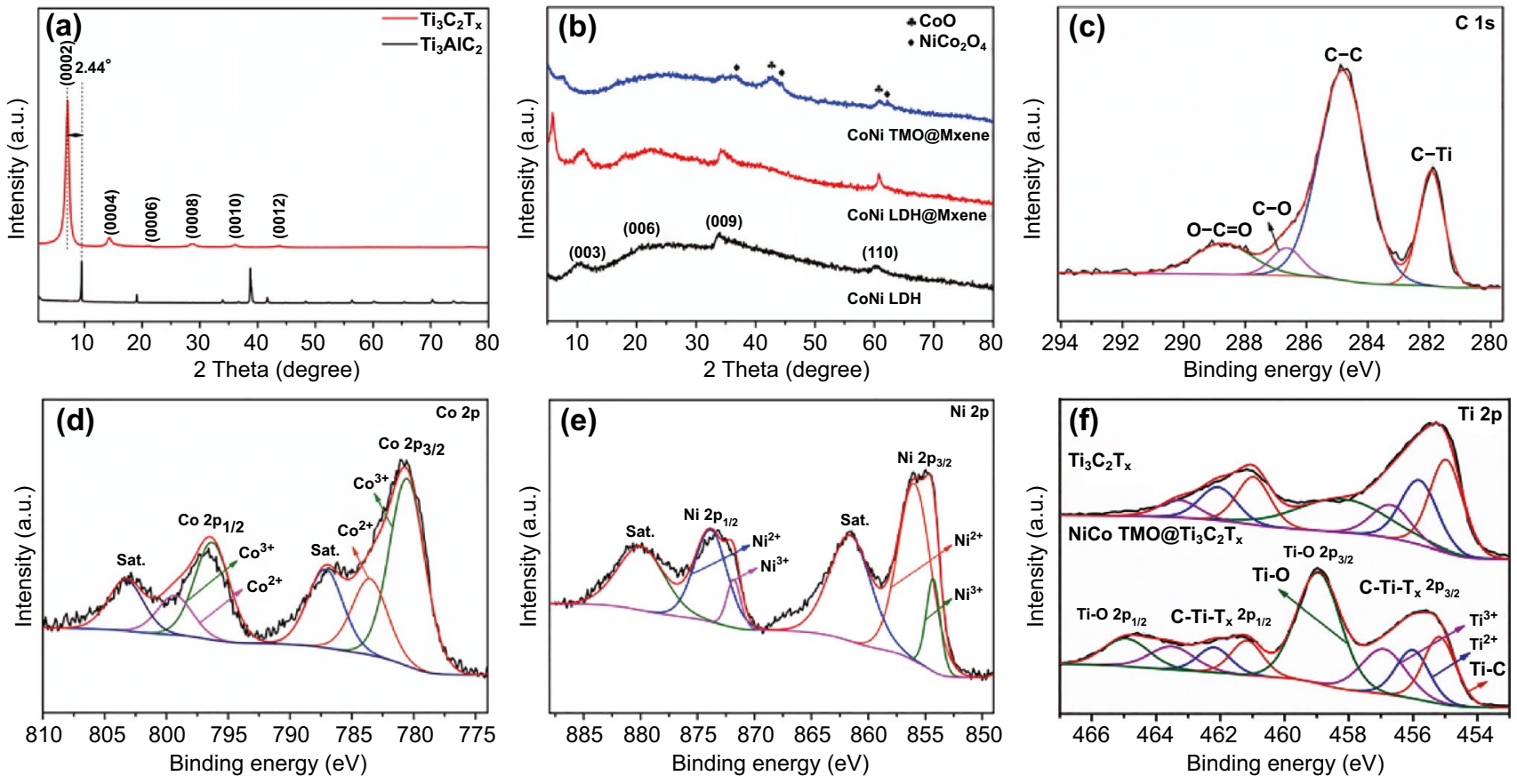

Fig. 5 a, b XRD patterns of as-prepared samples, $\mathbf{c} \mathrm{C}$ 1s, $\mathbf{d}$ Co 2p, e Ni 2p, and $\mathbf{f}$ Ti 2p spectra of TMOT and $\mathrm{Ti}_{3} \mathrm{C}_{2} \mathrm{~T}_{x}$

The XPS is also conducted to prove the elemental composition and valence states information. It reveals that TMOT composites mainly comprise $\mathrm{C}, \mathrm{Ti}, \mathrm{O}, \mathrm{F}, \mathrm{Co}$, and $\mathrm{Ni}$ elements in the survey spectrum (Fig. S4a), confirming the combination between NiCo TMO and $\mathrm{Ti}_{3} \mathrm{C}_{2} \mathrm{~T}_{x}$ MXene nanosheets. Figure $5 \mathrm{c}$ exhibits that the binding energy of $\mathrm{C}$ $1 \mathrm{~s}$ at 288.71, 286.64, 284.82, and 281.92 could be assigned to $\mathrm{O}-\mathrm{C}=\mathrm{O}, \mathrm{C}-\mathrm{O}, \mathrm{C}-\mathrm{C}$, and $\mathrm{C}-\mathrm{Ti}$ of TMOT composites, respectively. The deconvolution of Co 2 p spectrum (Fig. 5d) comprises two spin dipole orbitals (named Co $2 \mathrm{p}_{3 / 2}$, Co $2 \mathrm{p}_{1 / 2}$ ) and two shakeup satellites. The peaks at binding energy of 783.59 and $799.38 \mathrm{eV}$ belong to $\mathrm{Co}^{2+} 2 \mathrm{p}_{3 / 2}$ and
$2 \mathrm{p}_{1 / 2}$, while the peaks stood for $\mathrm{Co}^{3+} 2 \mathrm{p}_{3 / 2}$ and $2 \mathrm{p}_{1 / 2}$ present at 780.54 and $796.33 \mathrm{eV}$ [41]. Analogously, the Ni 2p spectrum for TMOT composites (Fig. 5e) also exhibits two spin-orbit doublets. And the fitting peaks at approximately $873.85,855.9,871.82$, and $854.31 \mathrm{eV}$ are in agreement with $\mathrm{Ni}^{2+} 2 \mathrm{p}_{1 / 2}, \mathrm{Ni}^{2+} 2 \mathrm{p}_{3 / 2}, \mathrm{Ni}^{3+} 2 \mathrm{p}_{1 / 2}$, and $\mathrm{Ni}^{3+} 2 \mathrm{p}_{3 / 2}$, apart from the noticeable satellite peaks at 861.60 and $879.96 \mathrm{eV}$ [42]. As shown in Fig. 5f, the Ti $2 p$ relates four peaks of TMOT composites at $455.1,455.9,456.9$, and $459.1 \mathrm{eV}$ corresponding to $\mathrm{Ti}-\mathrm{C}, \mathrm{Ti}^{2+}, \mathrm{Ti}^{3+}$, and $\mathrm{Ti}-\mathrm{O}$, respectively [43]. The percentage of Ti-O bond increases from $4.28 \%$ in the $\mathrm{Ti}_{3} \mathrm{C}_{2} \mathrm{~T}_{x}$ MXene to $41.54 \%$ in the TMOT, whereas no diffraction peak 
can be assigned to $\mathrm{TiO}_{2}$ in XRD pattern. It implies that the successful combination of $\mathrm{Ti}_{3} \mathrm{C}_{2} \mathrm{~T}_{x}$ MXene and NiCo TMO is formed through strong covalent bond interaction [44]. Compared to the LDHT sample (Fig. S4f), the C-Ti-T $x$ content of TMOT composites decreases due to disappearance of surface functional groups during annealing process.

The nitrogen adsorption-desorption isotherms are generally employed to characterize the Brunauer-Emmett-Teller (BET) surface area and pore structures of as-obtained samples. The isotherm of all samples (Fig. S5) could be categorized as type IV with a distinct $\mathrm{H} 3$ hysteresis loop, which is considered to be a slit hole formed by the accumulation of lamellar particles. It is consistent with the SEM and TEM images of the sample. Furthermore, the pore size distribution of LDH, LDHT and TMOT composites is mainly at $\approx$ $2 \mathrm{~nm}$. Plenty of pores can not only optimize the impedance matching, but also increase the flake-air interface, resulting in strong space charge polarization. The BET specific surface area of LDHT and TMOT composites calculated to be 83.94 and $68.47 \mathrm{~m}^{2} \mathrm{~g}^{-1}$ are larger than that of $\mathrm{Ti}_{3} \mathrm{C}_{2} \mathrm{~T}_{x}$ $\left(20.36 \mathrm{~m}^{2} \mathrm{~g}^{-1}\right)$, which may effectively trap microwaves and aggregate charges in abundant interfaces leading to interfacial polarization as well as related relaxation.

\subsection{Microwave Absorbing Property of LDHT and TMOT}

To realize the EM wave absorption properties of the LDHT and TMOT composites to the utmost extent, PVDF with unique dielectric property was chosen as the matrix. A dipole will be generated in the PVDF molecular chain derived from the different electronegativity of the $\mathrm{H}$ atom and the $\mathrm{F}$ atom, which will generate an electric dipole moment as well as dipole polarization under the action of an applied electric field [45]. As well, it is beneficial to construct flexible absorbers due to the inherent flexibility and wear resistance of PVDF (Fig. S6). As known, the MA property is strongly determined by its EM parameters, the complex relative permittivity $\left(\varepsilon_{r}, \varepsilon_{r}=\varepsilon^{\prime}-j \varepsilon^{\prime \prime}\right)$ and permeability $\left(\mu_{r}, \mu_{r}=\mu^{\prime}-j \mu^{\prime \prime}\right)$ [46]. Hence, the electromagnetic parameters of various samples with the PVDF matrix were measured over the range of $2-18 \mathrm{GHz}$, and the data are demonstrated in Figs. S7 and S8. For the $\mathrm{Ti}_{3} \mathrm{C}_{2} \mathrm{~T}_{x}$ sample, the values of $\varepsilon^{\prime}$ and $\varepsilon^{\prime \prime}$ are almost higher than those of the other samples over the whole frequency range. Obviously, with the increase in compound LDH content and decrease in filler loading (LDHT-x) in PVDF, both $\varepsilon^{\prime}$ and $\varepsilon^{\prime \prime}$ values are significantly reduced and deliver a similar declining trend with increasing frequency (Fig. S7). It manifests that the hyperactive conductivity of pure $\mathrm{Ti}_{3} \mathrm{C}_{2} \mathrm{~T}_{x}$ MXene can be balanced by coupling with hollow $\mathrm{LDH}$ nanocages, which also optimizes the impedance matching and ultimately influences MA performance. One can see that two peaks appeared in the $\varepsilon^{\prime \prime}-f$ curves of $\mathrm{Ti}_{3} \mathrm{C}_{2} \mathrm{~T}_{x}$ and LDHT-x composites, indicating multiple resonant properties (Fig. S7b, e). A heterojunction capacitor will form in the interface between LDH and $\mathrm{Ti}_{3} \mathrm{C}_{2} \mathrm{~T}_{x}$ MXene, which is conducive to the formation of resonance peak at $\sim 15 \mathrm{GHz}$ and generate more interfacial polarization [17]. Meanwhile, the dielectric loss tangent $\left(\tan \delta_{\varepsilon}=\varepsilon^{\prime \prime} / \varepsilon^{\prime}\right)$ and magnetic loss tangent $\left(\tan \delta_{\mu}=\mu^{\prime \prime} / \mu^{\prime}\right)$ related with the ability to convert EM energy were calculated to evaluate the microwave attenuation properties. The $\tan \delta_{\varepsilon}$ values of LDHT-x and $\mathrm{Ti}_{3} \mathrm{C}_{2} \mathrm{~T}_{x}$ MXene exhibit similar trends with the $\varepsilon^{\prime \prime}$ and remain higher value about $15 \mathrm{GHz}$ (Fig. S7c, f). Compared to changing LDHT-18 content in the PVDF, regulating the adherent $\mathrm{Ti}_{3} \mathrm{C}_{2} \mathrm{~T}_{x}$ attachment can adjust the $\varepsilon_{r}$ and $\tan \delta_{\varepsilon}$ value in extremely slight way. By increasing the LDHT-18 content, massive interfaces including LDHT-18/PVDF, LDH/ $\mathrm{Ti}_{3} \mathrm{C}_{2} \mathrm{~T}_{x}$ and LDHT-18/LDHT18 can serve as micro-capacitors with large capacity to store electric charge and enable high permittivity. In view of the non-magnetic property of the prepared material, the values of $\mu^{\prime}$ and $\mu^{\prime \prime}$ (Fig. S8) are almost constant and close to 1 and 0 , respectively. Importantly, LDHT-x composites possess higher dielectric loss values than magnetic loss, illustrating the dielectric loss plays a predominant role in the microwave attenuation performance.

Notably, dielectric loss inherently depends on conduction loss, multi-scattering and polarization relaxation that mainly arises from interface and dipole polarization [47, 48]. Ordinarily, the Debye dipolar relaxation process is conducted to reveal the mechanism of dielectric loss. According to Debye relaxation theory, the relationship between $\varepsilon$ ' and $\varepsilon$ ' can be expressed as [3]:

$\left(\varepsilon^{\prime}-\frac{\varepsilon_{s}+\varepsilon_{\infty}}{2}\right)^{2}+\left(\varepsilon^{\prime \prime}\right)^{2}=\left(\frac{\varepsilon_{s}-\varepsilon_{\infty}}{2}\right)^{2}$

where $\varepsilon_{s}$ and $\varepsilon_{\infty}$ represent static dielectric permittivity and the relative permittivity at an infinite frequency, respectively. It can be concluded that the $\varepsilon^{\prime}$ and $\varepsilon$ ', curves can form a semicircle marked as Cole-Cole semicircle, and 
each semicircle reveals a Debye relaxation process. Obviously, multiple twisted Cole-Cole semicircles are observed in 2-18 GHz from hollow LDHT-x/PVDF composites (Fig. S9c-f), authenticating the multiple polarization relaxation behaviors. These relaxation processes include the dipole polarization of surface groups on $\mathrm{Ti}_{3} \mathrm{C}_{2} \mathrm{~T}_{x}$, bonding charge and interface polarization induced by abundant heterogeneous interfaces and the porous hollow structure.

Reflection loss (RL) is calculated to evaluate intuitively the EM wave absorption abilities, which is in line with the transmission line theory. The formula is described as eq. [49]:

$\mathrm{RL}=20 \lg \left|\frac{Z_{\text {in }}-Z_{0}}{Z_{\text {in }}+Z_{0}}\right|$

$Z_{\text {in }}=Z_{0} \sqrt{\frac{\mu_{r}}{\varepsilon_{r}} \tanh \left(j \frac{2 \pi f d}{c} \sqrt{\mu_{r} \varepsilon_{r}}\right)}$

where $Z_{\text {in }}$ and $Z_{0}$ are is the input impedance of the absorber and free space, respectively. $f$ is the frequency of EM wave, $c$ is the light velocity in the free space, and $d$ is the thickness of the absorbing layers, respectively.

As for the laminar $\mathrm{Ti}_{3} \mathrm{C}_{2} \mathrm{~T}_{x}$ MXene shown in Fig. 6a, it merely exhibits negligible microwave absorption capacity with a minimum reflection loss value of $-15.68 \mathrm{~dB}$, which is originated from the incompatibility between high conductivity and impedance matching. Similarly, unsatisfied $\mathrm{RL}_{\text {min }}$ value of $-8.61 \mathrm{~dB}$ is also emerged in porous NiCo LDH with hollow structure (Fig. 6b). On account of the effective synergies among hollow hierarchical architectures as well as multi-components of $\mathrm{Ti}_{3} \mathrm{C}_{2} \mathrm{~T}_{x}$ MXene and porous NiCo LDH nanoparticles, LDHT-x realizes better MA properties than that of $\mathrm{Ti}_{3} \mathrm{C}_{2} \mathrm{~T}_{x}$ MXene and $\mathrm{LDH}$ (Fig. 6c-f). Particularly, LDHT-9 absorber (Fig. 6c) displays the $\mathrm{RL}_{\text {min }}$ value of $-36.88 \mathrm{~dB}$ and remarkably achieves an unexpected effective absorption bandwidth of $6.72 \mathrm{GHz}$ at only $2.10 \mathrm{~mm}$, covering the entire Ku-band. Specifically, LDHT-18 absorber represents the outstanding MA ability with $\mathrm{RL}_{\text {min }}$ value of $-40.84 \mathrm{~dB}$ and wide $\mathrm{EAB}$ of $3.28 \mathrm{GHz}$ covering almost $82 \%$ of the $\mathrm{X}$-band (Figs. 6e and S11). It is clearly observed that the filler loading of LDHT-x in PVDF has distinctly regulative effect on the MA performance (Fig. $\mathrm{S} 10)$. Besides, the optimal filler content is $15 \mathrm{wt} \%$. In conclude, adjusting the attachment $\mathrm{Ti}_{3} \mathrm{C}_{2} \mathrm{~T}_{x}$ flakes can subtly regulate the absorbing property with tune, the lowest $\mathrm{RL}_{\text {min }}$ and their corresponding frequency band. This phenomenon is consistent with the variation of electromagnetic parameters. Furthermore, the $\mathrm{RL}_{\text {min }}$ shifts to lower frequency accompanied by a reduction in EAB as increasing $\mathrm{Ti}_{3} \mathrm{C}_{2} \mathrm{~T}_{x}$ nanoflakes decoration at the same thickness of $2.5 \mathrm{~mm}$ with (a)
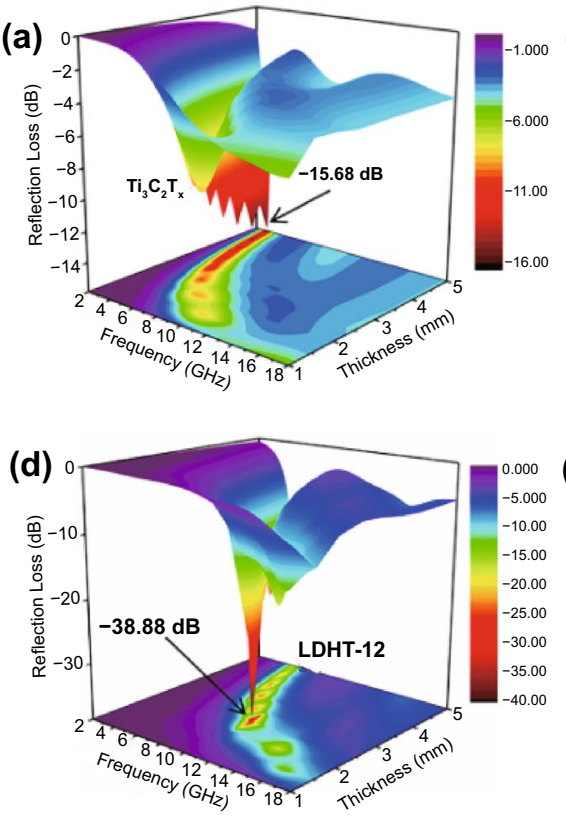

(b)

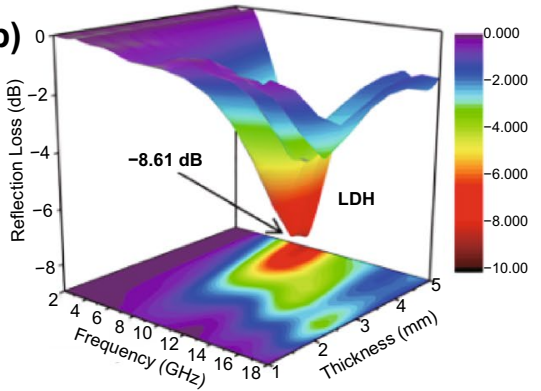

(e)

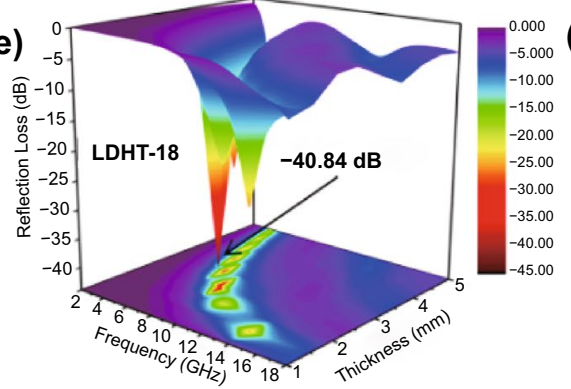

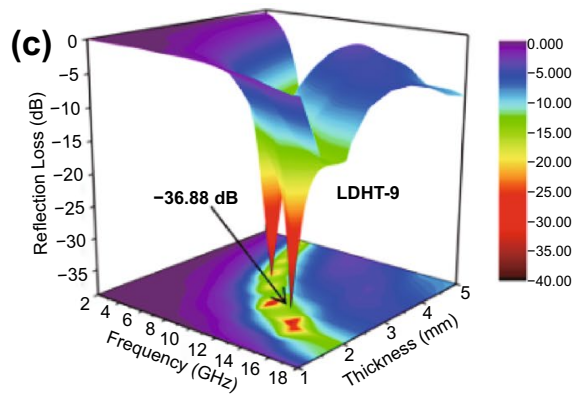

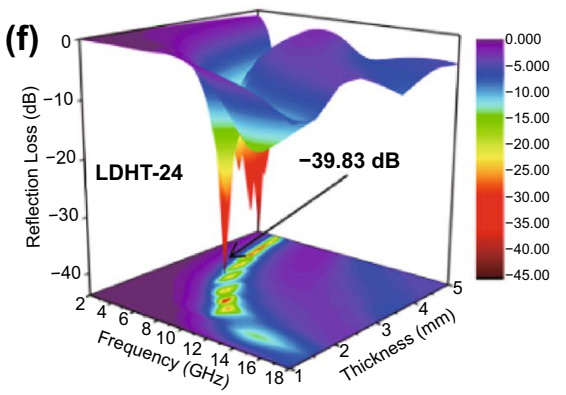

Fig. 6 3D RL values of $\mathbf{a} \mathrm{Ti}_{3} \mathrm{C}_{2} \mathrm{~T}_{x}$, b LDH, $\mathbf{c}$ LDHT-9, $\mathbf{d}$ LDHT-12, e LDHT-18 and $\mathbf{f}$ LDHT-24 composites with a filler content of 15 wt\% at different thicknesses 

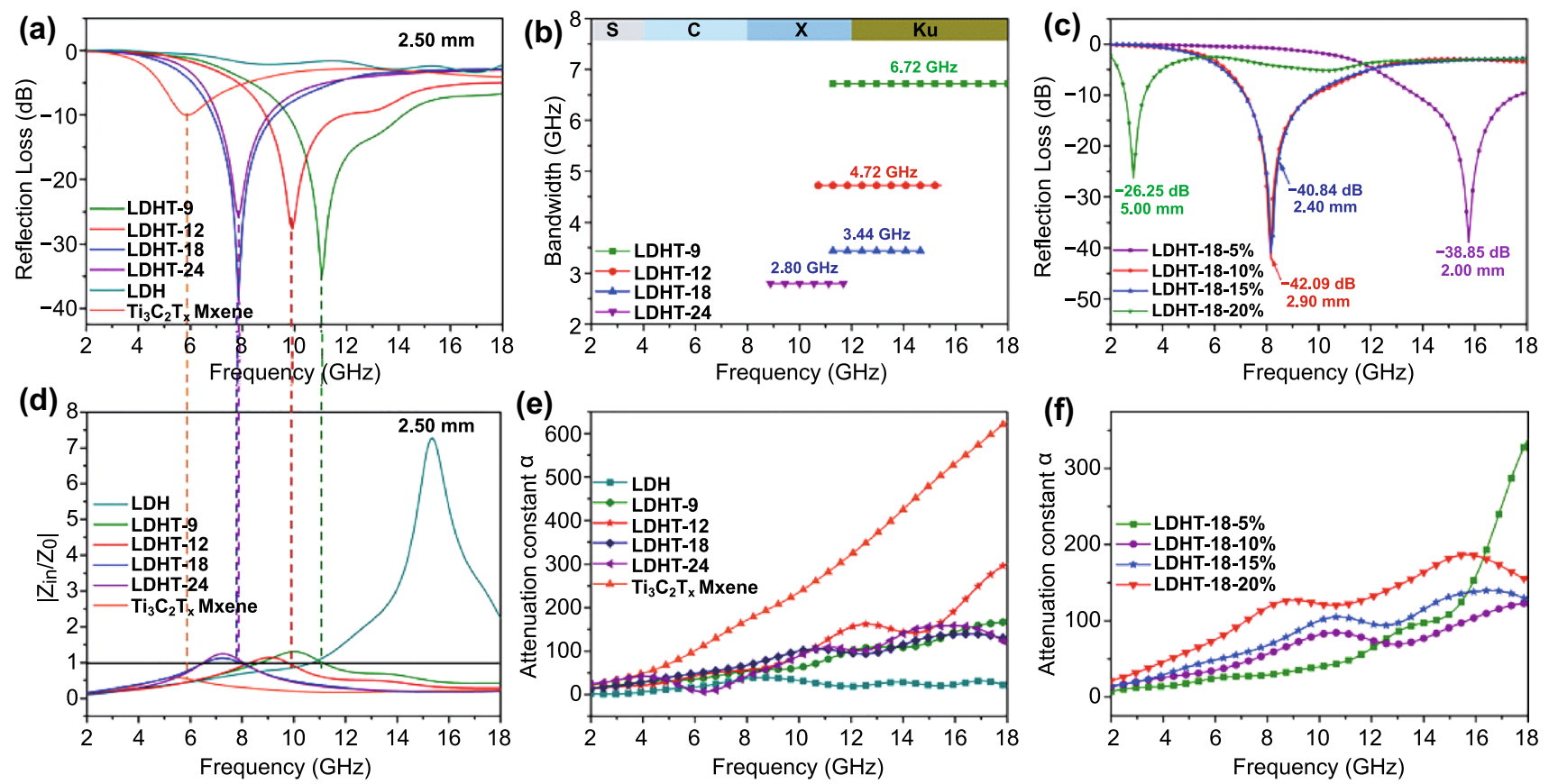

Fig. 7 a 2D curves of RL at $2.50 \mathrm{~mm}$, b EAB with the filler content of $15 \mathrm{wt} \%$. c RL curves at different loading in PVDF. d characteristic impedance curves, $\mathbf{e}$ attenuation constant of as-prepared samples in $15 \mathrm{wt} \%$. $\mathbf{f}$ attenuation constant at different loading in PVDF

the filling content of $15 \mathrm{wt} \%$ (Fig. 7a, b). Obviously, the optimal $\mathrm{RL}_{\min }$ value of LDHT-18 also follows this pattern (Fig. 7c), and this reminds us that changing filler content and $\mathrm{Ti}_{3} \mathrm{C}_{2} \mathrm{~T}_{x}$ MXene attachment are available to obtain a tunable and high-efficient microwave absorption material with broaden $\mathrm{EAB}$ and thin thickness.

To clearly clarify the microwave absorption enhancement mechanism of LDHT- $x$ /PVDF composites, the normalized characteristic impedance $\left(Z=\left|Z_{\text {in }}\right| Z_{0} \mid\right)$, which determines whether the electromagnetic wave can enter the material, and attenuation constant $(\alpha)$ related to comprehensive loss competence of absorbers are shown in Fig. 7d-f. Among them, the attenuation constant $(\alpha)$ can be figured out by equation [50]:

$\alpha=\frac{\sqrt{2} \pi f}{c} \sqrt{\left(\mu^{\prime \prime} \varepsilon^{\prime \prime}-\mu^{\prime} \varepsilon^{\prime}\right)+\sqrt{\left(\mu^{\prime \prime} \varepsilon^{\prime \prime}-\mu^{\prime} \varepsilon^{\prime}\right)^{2}+\left(\mu^{\prime} \varepsilon^{\prime \prime}+\mu^{\prime \prime} \varepsilon^{\prime}\right)^{2}}}$

As known, the impedance matching is the primary consideration for the designs of excellent EM wave absorber [51]. Generally, the impedance matching is greatly affected by the complex permittivity and the complex permeability $[52,53]$. Thus, reasonable regulation of EM parameter through adjusting the content of compound LDH and filler loading of LDHT-x in PVDF could be effective in achieving impedance matching. As shown in Fig. $7 \mathrm{~d}$, the $Z$ results reveal that the LDHT-x with loading of $15 \mathrm{wt} \%$ in PVDF, especially for LDHT-18 composites, obtain the improved impedance matching with more regions closing to or equaling 1 compared with individual $\mathrm{LDH}$ and $\mathrm{Ti}_{3} \mathrm{C}_{2} \mathrm{~T}_{x}$ MXene. In this case, LDHT-x will allow as much radiated EM wave as possible to enter its interior, paving the way for the subsequent consumption and absorption. Meanwhile, the peak of $\mathrm{Z}$ values shifts to the lower frequency as increasing decorated of $\mathrm{Ti}_{3} \mathrm{C}_{2} \mathrm{~T}_{x}$ MXene, which are consistent with the $\mathrm{RL}_{\min }$ in Fig. 6a. The attenuation constant $(\alpha)$ is another key factor to determine the EM absorbing performance as shown in Fig. 7e, f. The LDH sample displays the lowest $\alpha$ value, which reveals the weakest attenuation ability to incident microwaves. And the $\alpha$ values of LDHT-x composited are significantly enhanced after combining with $\mathrm{Ti}_{3} \mathrm{C}_{2} \mathrm{~T}_{x}$ MXene, and show a slight fluctuation as regulating $\mathrm{Ti}_{3} \mathrm{C}_{2} \mathrm{~T}_{x}$ MXene loading. Figure $7 \mathrm{f}$ further illustrates the effects of the different filler loadings on attenuation ability. The values of $\alpha$ are distinctly improved with increasing LDHT-18 loading from 5 to $20 \mathrm{wt} \%$. This is also in agreement with the results of EM parameters and refection loss. 
In accordance with the above findings, the EM parameter and MA ability of LDHT-x can be slightly adjusted by regulating the adherent $\mathrm{Ti}_{3} \mathrm{C}_{2} \mathrm{~T}_{x}$ nanoflakes amount. Therefore, TMOT-x hollow architectures with more compound proportions were prepared by pyrolyzing the corresponding LDHT-x composites to further improve the microwave attenuation capacity. It is obvious that TMOT-x absorbers possess remarkably enhanced EM absorbing properties with lower $\mathrm{RL}_{\min }$ and filler content in PVDF compared with the LDHT composite (Figs. 8 and S12). Besides, the MA performance of TMOT-x absorbers are not desirable when the filler loading increases to $15 \mathrm{wt} \%$, which may be attributed to high-frequency oscillating skin current and reflection of incident microwave caused by excessive $3 \mathrm{D}$ conductive network $[15,54]$. The absorption peak gradually moves to the low frequency and low filling amount with increasing the $\mathrm{Ti}_{3} \mathrm{C}_{2} \mathrm{~T}_{x}$ MXene attachment. To be specific, as shown in Fig. 8a, TMOT-12 absorber could achieve excellent MA properties with the reflection loss of $-50.37 \mathrm{~dB}$ and the corresponding $\mathrm{EAB}$ of $3.44 \mathrm{GHz}(7.12-10.56 \mathrm{GHz})$ at $3.20 \mathrm{~mm}$. When the volume of $\mathrm{Ti}_{3} \mathrm{C}_{2} \mathrm{~T}_{x}$ MXene suspension increases to $15 \mathrm{~mL}$, the effective EM absorption occurs at lower filling mass fraction of $5 \mathrm{wt} \%$ (Fig. S12). And the optimal filler content is trended toward $5 \mathrm{wt} \%$ as increasing continuously the anchored $\mathrm{Ti}_{3} \mathrm{C}_{2} \mathrm{~T}_{x}$ MXene flake. Furthermore, TMOT-18 absorber exhibit $\mathrm{RL}_{\min }$ of -43.22 and $-46.30 \mathrm{~dB}$ at 1.40 and $2.20 \mathrm{~mm}$, respectively (Fig. 8c). Surprisingly, for TMOT21 absorber as shown in Fig. 8d, it remarkably emerges an unexpected $\mathrm{RL}_{\min }$ value of $-67.22 \mathrm{~dB}$ corresponding to the absorption bandwidth of $3.84 \mathrm{GHz}$ at $1.70 \mathrm{~mm}$. When the thickness changes to $4.00 \mathrm{~mm}$, the $\mathrm{RL}_{\text {min }}$ value reaches $-48.37 \mathrm{~dB}$ at low frequency $(5.6 \mathrm{GHz})$ within only $5 \mathrm{wt} \%$ filler loading. Thus, TMOT-21 absorber equips with absolute advantages on light weight and ultrathin thickness, and realizes splendid MA performance with remarkable $\mathrm{RL}_{\min }$ and tunable EAB.

The underlying MA mechanisms of the unique 3D hollow hierarchical NiCo compound@MXene composites are shown in Fig. 9. The hollow layered structure with open void space will optimize impedance matching behavior and contribute to lightweight absorber as well as generate more interfacial polarization [55]. Besides, bending $\mathrm{Ti}_{3} \mathrm{C}_{2} \mathrm{~T}_{x}$ MXene sheets with random orientation is beneficial to construct $3 \mathrm{D}$ conductive network to enable free migration of electrons in conductive pathways, which would further enhance the conduction loss to consume the EM wave by converting EM energy into thermal energy [56]. Simultaneously, 3D porous hierarchical NiCo compound@MXene composites can provide multiple reflection and scattering to prolong the transmission path of electromagnetic waves, leading to the dissipation of electromagnetic


Fig. 8 3D plots of RL values for various TMOT-x composites 




Fig. 9 Schematic illustration of microwave absorption mechanism for TMOT

energy [43, 53]. Meanwhile, substantial termination functional groups and intrinsic defects of layered $\mathrm{Ti}_{3} \mathrm{C}_{2} \mathrm{~T}_{x}$ MXene as well as the oxygen vacancies of $\mathrm{NiCo} \mathrm{TMO} / \mathrm{LDH}$ caused by calcination effectively optimize the impedance matching, while giving rise to the enhanced dipole polarization and defect polarization. The 3D hierarchical structure assembled by $\mathrm{Ti}_{3} \mathrm{C}_{2} \mathrm{~T}_{x}$ MXene flakes and hollow NiCo TMO/LDH can offer high density of heterogeneous interfaces, which will induce accumulation and uneven distribution of space charges and produce a macroscopic electric moment leading to remarkable interfacial polarization (called as the Maxwell-Wagner effect) [57].

Finally, the optimized RL values $\left(\mathrm{ORL}_{1}\right)$ are introduced to reasonably evaluate MA ability as considering filler loading [58]:

$\mathrm{ORL}_{1}=\frac{\mathrm{RL}}{\text { filler loading }}$

Compared with other works, a desirable effective absorption bandwidth of LDHT-9 absorber is up to $6.72 \mathrm{GHz}$ with the thickness of $2.10 \mathrm{~mm}$, which can be comparable to the progressive absorbing material (Fig. 10). It is worth noting that TMOT-21 absorber achieves inspiring MA performance with the $\mathrm{RL}_{\min }$ value of $-67.22 \mathrm{~dB}$ and $\mathrm{EAB}$ of $3.84 \mathrm{GHz}$ at $1.70 \mathrm{~mm}$ under extremely low filling content of $5 \mathrm{wt} \%$. And the $\mathrm{ORL}_{1}$ value of TMOT-21 absorber almost surpasses various kinds of advanced absorbents reported by previous literatures, which is closer to the ideal absorbing material.

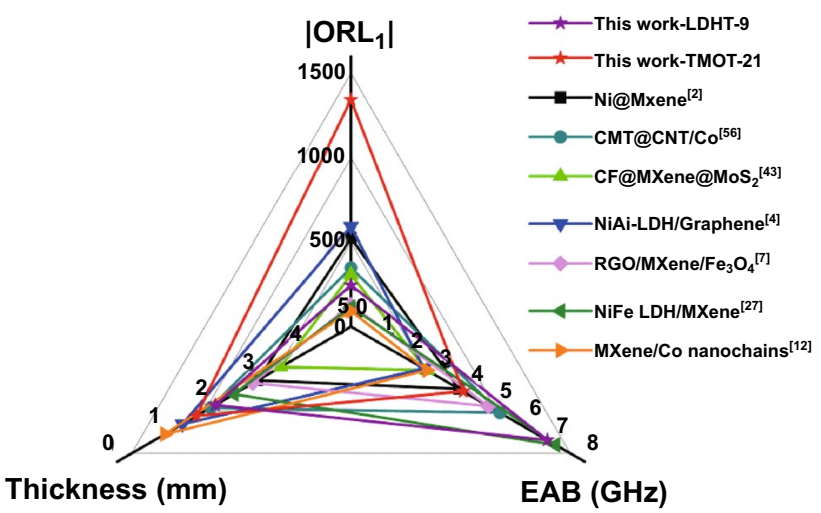

Fig. 10 Comparison of the MA properties of various related materials

\section{Conclusion}

In summary, the unique 3D NiCo compound@MXene that inherited the hollow polyhedral skeleton structure and excellent conductive network has been successfully synthesized by facile electrostatically assembling method and calcination treatment. The formation of hierarchical architecture efficiently prevents the restacking of $2 \mathrm{D} \mathrm{Ti}_{3} \mathrm{C}_{2} \mathrm{~T}_{x}$ nanosheets and further enlarges the specific surface area which is beneficial to the conduction loss and polarization. It is concluded that the EM parameters and MA performance can be regulated by the adherent $\mathrm{Ti}_{3} \mathrm{C}_{2} \mathrm{~T}_{x}$ content and filler loading amount in PVDF matrix. The LDHT-9 remarkably possesses ultra-wide EAB of $6.72 \mathrm{GHz}$ at the thickness of $2.10 \mathrm{~mm}$. The TMOT21 near the percolation threshold gives rise to brilliant EM absorbing ability with an optimal $\mathrm{RL}_{\min }$ value of $-67.22 \mathrm{~dB}$ and $\mathrm{EAB}$ of $3.84 \mathrm{GHz}$ at $1.70 \mathrm{~mm}$ with only $5 \mathrm{wt} \%$ loading. Besides, the combination of the hybrids and PVDF endow the absorber with flexibility and wear resistance as well. Thus, this work provides inspiration to construct 3D hollow hierarchical structure and efficiently moderate electromagnetic parameters, which might help for promising candidate in ultralight and ultrathin MA absorbers.

Acknowledgements This work was supported by the National Natural Science Foundation of China (No. 52073010) and Beijing Natural Science Foundation (2214069).

Open Access This article is licensed under a Creative Commons Attribution 4.0 International License, which permits use, sharing, adaptation, distribution and reproduction in any medium or format, as long as you give appropriate credit to the original author(s) and the source, provide a link to the Creative Commons licence, and 
indicate if changes were made. The images or other third party material in this article are included in the article's Creative Commons licence, unless indicated otherwise in a credit line to the material. If material is not included in the article's Creative Commons licence and your intended use is not permitted by statutory regulation or exceeds the permitted use, you will need to obtain permission directly from the copyright holder. To view a copy of this licence, visit http://creativecommons.org/licenses/by/4.0/.

Supplementary Information The online version contains supplementary material available at https://doi.org/10.1007/s40820-02100727-y.

\section{References}

1. Z.H. Zhao, K.C. Kou, L.M. Zhang, H.J. Wu, High efficiency electromagnetic wave absorber derived from transition metal layered double hydroxides. J. Colloid Interface Sci. 579, 733740 (2020). https://doi.org/10.1016/j.jcis.2020.06.123

2. L.Y. Liang, R.S. Yang, G.J. Han, Y.Z. Feng, B. Zhao et al., Enhanced electromagnetic wave-absorbing performance of magnetic nanoparticles-anchored $2 \mathrm{D} \mathrm{Ti}_{3} \mathrm{C}_{2} \mathrm{~T}_{\mathrm{x}}$ MXene. ACS Appl. Mater. Interfaces 12, 2644-2654 (2020). https://doi.org/ 10.1021/acsami.9b18504

3. X.C. Di, Y. Wang, Y.Q. Fu, X.M. Wu, P. Wang, Wheat flourderived nanoporous carbon@ $\mathrm{ZnFe}_{2} \mathrm{O}_{4}$ hierarchical composite as an outstanding microwave absorber. Carbon 173, 174-184 (2021). https://doi.org/10.1016/j.carbon.2020.11.006

4. X.F. Xu, S.H. Shi, Y.L. Tang, G.Z. Wang, M.F. Zhou et al., Growth of NiAl-layered double hydroxide on graphene toward excellent anticorrosive microwave absorption application. Adv. Sci. 8, 2002658 (2021). https://doi.org/10.1002/advs.202002658

5. X.Y. Zhu, H.F. Qiu, P. Chen, G.Z. Chen, W.X. Min, Graphitic carbon nitride $\left(\mathrm{g}-\mathrm{C}_{3} \mathrm{~N}_{4}\right)$ in situ polymerization to synthesize MOF-Co@CNTs as efficient electromagnetic microwave absorption materials. Carbon 176, 530-539 (2021). https://doi. org/10.1016/j.carbon.2021.02.044

6. W.T. Cao, C. Ma, S. Tan, M.G. Ma, P.B. Wan et al., Ultrathin and flexible CNTs/MXene/Cellulose nanofibrils composite paper for electromagnetic interference shielding. Nano-Micro Lett. 11, 72 (2019). https://doi.org/10.1007/s40820-019-0304-y

7. Y.H. Cui, K. Yang, J.Q. Wang, T. Shah, Q.Y. Zhang et al., Preparation of pleated RGO/MXene/ $\mathrm{Fe}_{3} \mathrm{O}_{4}$ microsphere and its absorption properties for electromagnetic wave. Carbon 172, 1-14 (2021). https://doi.org/10.1016/j.carbon.2020.09.093

8. Y.Z. Zhang, J. El-Demellawi, Q. Jiang, G. Ge, H.F. Liang et al., MXene hydrogels: fundamentals and applications. Chem. Soc. Rev. 49, 7229-7251 (2020). https://doi.org/10.1039/D0CS0 0022A

9. L.Y. Liang, G.J. Han, Y. Li, B. Zhao, B. Zhou et al., Promising $\mathrm{Ti}_{3} \mathrm{C}_{2} \mathrm{~T}_{\mathrm{x}}$ MXene/Ni chain hybrid with excellent electromagnetic wave absorption and shielding capacity. ACS Appl. Mater. Interfaces 11, 25399-25409 (2019). https://doi.org/10. 1021/acsami.9b07294
10. Y. Liu, S. Zhang, X.L. Su, J. Xu, Y.Y. Li, Enhanced microwave absorption properties of $\mathrm{Ti}_{3} \mathrm{C}_{2}$ MXene powders decorated with Ni particles. J. Mater. Sci. 55, 10339-10350 (2020). https:// doi.org/10.1007/s10853-020-04739-8

11. Z.M. Fan, J.F. Wang, H. Kang, Y.S. Wang, Z.M. Xie et al., A Compact MXene film with folded structure for advanced supercapacitor electrode material. ACS Appl. Energy Mater. 3, 1811-1820 (2020). https://doi.org/10.1021/acsaem.9b022 59

12. F. Pan, L.Z. Yu, Z. Xiang, Z.C. Liu, B.W. Deng et al., Improved synergistic effect for achieving ultrathin microwave absorber of 1D Co nanochains/2D carbide MXene nanocomposite. Carbon 172, 506-515 (2021). https://doi.org/10.1016/j. carbon.2020.10.039

13. M.Q. Zhao, M. Torelli, C.E. Ren, M. Ghidiu, Z. Ling et al., 2D titanium carbide and transition metal oxides hybrid electrodes for Li-ion storage. Nano Energy 30, 603-613 (2016). https:// doi.org/10.1016/j.nanoen.2016.10.062

14. J. Liu, H.B. Zhang, X. Xie, R. Yang, Z.S. Liu et al., Multifunctional, superelastic, and lightweight MXene/polyimide aerogels. Small 14, 1-10 (2018). https://doi.org/10.1002/smll. 201802479

15. M.S. Cao, Y.Z. Cai, P. He, J.C. Shu, W.Q. Cao et al., 2D MXenes: electromagnetic property for microwave absorption and electromagnetic interference shielding. Chem. Eng. J. 359, 1265-1302 (2019). https://doi.org/10.1016/j.cej.2018.11.051

16. P. Zhang, Q.Z. Zhu, R. Soomro, S.Y. He, N. Sun et al., In situ ice template approach to fabricate 3D flexible MXene film-based electrode for high performance supercapacitors. Adv. Funct. Mater. 30, 2000922 (2020). https://doi.org/10.1002/adfm.20200 0922

17. X. Li, W.B. You, L. Wang, J.W. Liu, Z.C. Wu et al., Self-assembly-magnetized MXene avoid dual-agglomeration with enhanced interfaces for strong microwave absorption through a tunable electromagnetic property. ACS Appl. Mater. Interfaces 11, 44536-44544 (2019). https://doi.org/10.1021/acsami.9b11861

18. Y. Xia, T.S. Mathis, M.Q. Zhao, B. Anasori, A. Dang et al., Thickness-independent capacitance of vertically aligned liquidcrystalline MXenes. Nature 557, 409-412 (2018). https://doi. org/10.1038/s41586-018-0109-z

19. M.Q. Zhao, X.Q. Xie, C.E. Ren, T. Makaryan, B. Anasori et al., Hollow MXene spheres and 3D macroporous MXene frameworks for Na-ion storage. Adv. Mater. 29, 1702410 (2017). https://doi.org/10.1002/adma.201702410

20. K. Li, M.Y. Liang, H. Wang, X.H. Wang, Y.S. Huang et al., 3D MXene architectures for efficient energy storage and conversion. Adv. Funct. Mater. 30, 2000842 (2020). https://doi.org/10.1002/ adfm.202000842

21. M. Naguib, O. Mashtalir, M.R. Lukatskaya, B. Dyatkin, C.F. Zhang et al., One-step synthesis of nanocrystalline transition metal oxides on thin sheets of disordered graphitic carbon by oxidation of MXenes. Chem. Commun. 50, 7420-7423 (2014). https://doi.org/10.1039/c4cc01646g

22. D.W. Chu, F.B. Li, X.M. Song, H.Y. Ma, L.H. Tan et al., A novel dual-tasking hollow cube $\mathrm{NiFe}_{2} \mathrm{O}_{4}$-NiCo-LDH@ $\mathrm{rGO}$ hierarchical material for high preformance supercapacitor and 
glucose sensor. J. Colloid Interface Sci. 568, 130-138 (2020). https://doi.org/10.1016/j.jcis.2020.02.012

23. B. Wen, H.B. Yang, L. Wang, Y. Qiu, Hierarchical $\mathrm{Co}_{\mathrm{X}} \mathrm{Al}_{\mathrm{y}}$ layered double hydroxide@carbon composites derived from metal-organic frameworks with efficient broadband electromagnetic wave absorption. J. Mater. Chem. C 8, 16418-16426 (2020). https://doi.org/10.1039/d0tc00622j

24. R. Ramachandran, Y.C. Lan, Z.X. Xu, F. Wang, Construction of NiCo-layered double hydroxide microspheres from Ni-MOFs for high-performance asymmetric supercapacitors. ACS Appl. Energy Mater. 3, 6633-6643 (2020). https://doi. org/10.1021/acsaem.0c00790

25. Y. Xiong, L.L. Xu, C.X. Yang, Q.F. Sun, X.J. Xu, Implanting $\mathrm{FeCo} / \mathrm{C}$ nanocages with tunable electromagnetic parameters in anisotropic wood carbon aerogels for efficient microwave absorption. J. Mater. Chem. A 8, 18863-18871 (2020). https:// doi.org/10.1039/d0ta05540a

26. Y.Z. Zhao, W. Wang, J.N. Wang, J.J. Zhai, X.Y. Lei et al., Constructing multiple heterogeneous interfaces in the composite of bimetallic MOF-derivatives and rGO for excellent microwave absorption performance. Carbon 173, 1059-1072 (2020). https://doi.org/10.1016/j.carbon.2020.11.090

27. Z.H. Wang, L.X. Yang, Y. Zhou, C. Xu, M. Yan et al., NiFe $\mathrm{LDH} / \mathrm{MXene}$ derivatives interconnected with carbon fabric for flexible electromagnetic wave absorption. ACS Appl. Mater. Interfaces 13, 16713-16721 (2021). https://doi.org/10.1021/ acsami.1c05007

28. Y.L. Wang, S.H. Yang, H.Y. Wang, G.S. Wang, X.B. Sun et al., Hollow porous $\mathrm{CoNi} / \mathrm{C}$ composite nanomaterials derived from MOFs for efficient and lightweight electromagnetic wave absorber. Carbon 167, 485-494 (2020). https://doi.org/10. 1016/j.carbon.2020.06.014

29. Y. Feng, D. Xiang, Y. Qiu, L. Li, Y. Li et al., MOF-derived spinel $\mathrm{NiCo}_{2} \mathrm{O}_{4}$ hollow nanocages for the construction of nonenzymatic electrochemical glucose sensor. Electroanalysis 32, 571-580 (2020). https://doi.org/10.1002/elan.201900558

30. X. Zhou, X.W. Mu, W. Cai, J.L. Wang, F.K. Chu et al., Design of hierarchical NiCo-LDH@PZS hollow dodecahedron architecture and application in high-performance epoxy resin with excellent fire safety. ACS Appl. Mater. Interfaces 11, 41736-41749 (2019). https://doi.org/10.1021/acsami.9b16482

31. Y. Li, F.B. Meng, Y. Mei, H.G. Wang, Y.F. Guo et al., Electrospun generation of $\mathrm{Ti}_{3} \mathrm{C}_{2} \mathrm{~T}_{\mathrm{x}}$ MXene@ graphene oxide hybrid aerogel microspheres for tunable high-performance microwave absorption. Chem. Eng. J. 391, 123512 (2020). https://doi.org/ 10.1016/j.cej.2019.123512

32. X.Q. Cai, X.P. Shen, L.B. Ma, Z.Y. Ji, C. Xu et al., Solvothermal synthesis of NiCo-layered double hydroxide nanosheets decorated on RGO sheets for high performance supercapacitor. Chem. Eng. J. 268, 251-259 (2015). https://doi.org/10.1016/j. cej.2015.01.072

33. P. He, M.S. Cao, Y.Z. Cai, J.C. Shu, W.Q. Cao et al., Selfassembling flexible 2D carbide MXene film with tunable integrated electron migration and group relaxation toward energy storage and green EMI shielding. Carbon 157, 80-89 (2020). https://doi.org/10.1016/j.carbon.2019.10.009
34. J.W. Wen, X.X. Li, G. Chen, Z.N. Wang, X.J. Zhou et al., Controllable adjustment of cavity of core-shelled $\mathrm{Co}_{3} \mathrm{O}_{4} @$ $\mathrm{NiCo}_{2} \mathrm{O}_{4}$ composites via facile etching and deposition for electromagnetic wave absorption. J. Colloid Interface Sci. 594, 424-434 (2021). https://doi.org/10.1016/j.jcis.2021.03.056

35. Z.K. Jia, Y.S. Ma, L.Y. Yang, C.P. Guo, N. Zhou et al., $\mathrm{NiCo}_{2} \mathrm{O}_{4}$ spinel embedded with carbon nanotubes derived from bimetallic NiCo metal-organic framework for the ultrasensitive detection of human immune deficiency virus-1 gene. Biosens. Bioelectron. 133, 55-63 (2019). https://doi.org/10. 1016/j.bios.2019.03.030

36. M. Ghidiu, M.R. Lukatskaya, M.Q. Zhao, Y. Gogotsi, M.W. Barsoum, Conductive two-dimensional titanium carbide "clay" with high volumetric capacitance. Nature 516, 78-81 (2014). https://doi.org/10.1038/nature13970

37. M. Alhabeb, K. Maleski, B. Anasori, P. Lelyukh, L. Clark et al., Guidelines for synthesis and processing of two-dimensional titanium carbide $\left(\mathrm{Ti}_{3} \mathrm{C}_{2} \mathrm{~T}_{\mathrm{x}}\right.$ MXene). Chem. Mater. 29, 7633-7644 (2017). https://doi.org/10.1021/acs.chemmater. $7 \mathrm{~b} 02847$

38. J.F. Chen, Q. Ru, Y.D. Mo, S.J. Hu, X.H. Hou, Design and synthesis of hollow $\mathrm{NiCo}_{2} \mathrm{O}_{4}$ nanoboxes as anodes for lithiumion and sodium-ion batteries. Phys. Chem. Chem. Phys. 18, 18949-18957 (2016). https://doi.org/10.1039/c6cp02871c

39. J. Li, S. Lu, H.L. Huang, D.H. Liu, Z.B. Zhuang et al., ZIF-67 as continuous Self-Sacrifice template derived $\mathrm{NiCo}_{2} \mathrm{O}_{4} / \mathrm{Co}$, $\mathrm{N}-\mathrm{CNTs}$ nanocages as efficient bifunctional electrocatalysts for rechargeable Zn-Air batteries. ACS Sustain. Chem. Eng. 6, 10021-10029 (2018). https://doi.org/10.1021/acssuschem eng.8b01332

40. H.J. Hu, J.Y. Liu, Z.H. Xu, L.Y. Zhang, B. Cheng et al., Hierarchical porous $\mathrm{Ni} / \mathrm{Co}-\mathrm{LDH}$ hollow dodecahedron with excellent adsorption property for Congo red and Cr(VI) ions. Appl. Surf. Sci. 478, 981-990 (2019). https://doi.org/10.1016/j. apsusc.2019.02.008

41. W.Y. Chen, B. Han, Y.L. Xie, S.J. Liang, H. Deng et al., Ultrathin Co-Co LDHs nanosheets assembled vertically on MXene: 3D nanoarrays for boosted visible-light-driven $\mathrm{CO}_{2}$ reduction. Chem. Eng. J. 391, 123519 (2020). https://doi.org/ 10.1016/j.cej.2019.123519

42. D.D. Wang, S.D. Li, Y.C. Du, X.F. Wu, Y.F. Chen, Self-templating synthesis of $3 \mathrm{D}$ hierarchical $\mathrm{NiCo}_{2} \mathrm{O}_{4} @ \mathrm{NiO}$ nanocage from hydrotalcites for toluene oxidation. Catalysts 9, 352 (2019). https://doi.org/10.3390/catal9040352

43. J.Q. Wang, L. Liu, S.L. Jiao, K.J. Ma, J. Lv et al., Hierarchical carbon fiber@MXene@ $\mathrm{MoS}_{2}$ core-sheath synergistic microstructure for tunable and efficient microwave absorption. Adv. Funct. Mater. 30, 2002595 (2020). https://doi.org/10.1002/ adfm.202002595

44. R. Zhang, Z. Xue, J.Q. Qin, M. Sawangphruk, X.Y. Zhang et al., $\mathrm{NiCo}-\mathrm{LDH} / \mathrm{Ti}_{3} \mathrm{C}_{2} \mathrm{MXene}$ hybrid materials for lithium ion battery with high-rate capability and long cycle life. J. Energy Chem. 50, 143-153 (2020). https://doi.org/10.1016/j. jechem.2020.03.018

45. S. Gao, G.S. Wang, L. Guo, S.H. Yu, Tunable and ultraefficient microwave absorption properties of trace $\mathrm{N}$-doped 
two-dimensional carbon-based nanocomposites loaded with multi-rare earth oxides. Small 16, 1906668 (2020). https:// doi.org/10.1002/smll.201906668

46. F. Zhang, W. Cui, B.B. Wang, B.H. Xu, X.H. Liu et al., Morphology-control synthesis of polyaniline decorative porous carbon with remarkable electromagnetic wave absorption capabilities. Compos. Part B Eng. 204, 108491 (2021). https:// doi.org/10.1016/j.compositesb.2020.108491

47. Y.P. Zhao, H. Zhang, X. Yang, H. Huang, G.L. Zhao et al., In situ construction of hierarchical core-shell $\mathrm{Fe}_{3} \mathrm{O}_{4} @ \mathrm{C}$ nanoparticles-helical carbon nanocoil hybrid composites for highly efficient electromagnetic wave absorption. Carbon 171, 395-408 (2021). https://doi.org/10.1016/j.carbon.2020.09.036

48. Q. Song, F. Ye, L. Kong, Q.L. Shen, L.Y. Han et al., Graphene and MXene nanomaterials: toward high-performance electromagnetic wave absorption in gigahertz band range. Adv. Funct. Mater. 30, 2000475 (2020). https://doi.org/10.1002/ adfm. 202000475

49. Z.C. Wu, Z.Q. Yang, C. Jin, Y.H. Zhao, R.C. Che, Accurately engineering 2D/2D/0D heterojunction in hierarchical $\mathrm{Ti}_{3} \mathrm{C}_{2} \mathrm{~T}_{\mathrm{x}}$ MXene nanoarchitectures for electromagnetic wave absorption and shielding. ACS Appl. Mater. Interfaces 13, 5866-5876 (2021). https://doi.org/10.1021/acsami.0c21833

50. M.Q. Huang, L. Wang, K. Pei, W.B. You, X.F. Yu et al., Multidimension-controllable synthesis of MOF-derived Co@Ndoped carbon composite with magnetic-dielectric synergy toward strong microwave absorption. Small 16, 2000158 (2020). https://doi.org/10.1002/smll.202000158

51. D.Q. Zhang, T.T. Liu, J.Y. Cheng, Q. Cao, G.P. Zheng et al., Lightweight and high-performance microwave absorber based on $2 \mathrm{D} \mathrm{WS}_{2}-\mathrm{RGO}$ heterostructures. Nano-Micro Lett. 11, 38 (2019). https://doi.org/10.1007/s40820-019-0270-4

52. D.Q. Zhang, H.B. Zhang, J.Y. Cheng, H. Raza, T.T. Liu et al., Customizing coaxial stacking $\mathrm{VS}_{2}$ nanosheets for dual-band microwave absorption with superior performance in the $\mathrm{C}$ - and Ku- bands. J. Mater. Chem. C 8, 5923-5933 (2020). https:// doi.org/10.1039/D0TC00763C

53. J.Y. Cheng, H.B. Zhang, Y.F. Xiong, L.F. Gao, B. Wen et al., Construction of multiple interfaces and dielectric/magnetic heterostructures in electromagnetic wave absorbers with enhanced absorption performance: a review. J. Materiomics (2021). https://doi.org/10.1016/j.jmat.2021.02.017

54. Z. Wang, Z. Cheng, C.Q. Fang, X.L. Hou, L. Xie, Recent advances in MXenes composites for electromagnetic interference shielding and microwave absorption. Compos. Part A Appl. Sci. Manuf. 136, 105956 (2020). https://doi.org/10. 1016/j.compositesa.2020.105956

55. X.J. Zhang, J.Q. Zhu, P.G. Yin, A.P. Guo, A.P. Huang et al., Tunable high-performance microwave absorption of $\mathrm{Co}_{1-\mathrm{x}} \mathrm{S}$ hollow spheres constructed by nanosheets within ultralow filler loading. Adv. Funct. Mater. 28, 1800761 (2018). https:// doi.org/10.1002/adfm.201800761

56. Z.C. Wu, K. Pei, L.S. Xing, X.F. Yu, W.B. You et al., Enhanced microwave absorption performance from magnetic coupling of magnetic nanoparticles suspended within hierarchically tubular composite. Adv. Funct. Mater. 29, 1901448 (2019). https://doi.org/10.1002/adfm.201901448

57. Q.Q. Li, Y.H. Zhao, X.H. Li, L. Wang, X. Li et al., MOF induces $2 \mathrm{D} \mathrm{GO}$ to assemble into 3D accordion-Like composites for tunable and optimized microwave absorption performance. Small 16, 2003905 (2020). https://doi.org/10.1002/ smll.202003905

58. F. Wu, K. Yang, Q. Li, T. Shah, M. Ahmad et al., Biomassderived 3D magnetic porous carbon fibers with a helical/chiral structure toward superior microwave absorption. Carbon 173, 918-931 (2021). https://doi.org/10.1016/j.carbon.2020.11.088 